\title{
RFID als Feld für politische Debatten und Überzeugungsarbeit \\ Lobbying, politische Kommunikation und Themenmanagement
}

\section{Marco Althaus}

Politische Aspekte der RFID-Technologie werden im Bibliothekswesen überwiegend sektorspezifisch diskutiert. In der Vertretung ihrer Interessen gegenüber politischen Entscheidungsträgern stehen bibliothekstypische Finanzierungs- und Organisationsfragen im Vordergrund. Dabei kann jedoch die sektorübergreifende Kenntnis des Lobbyings von Industrie und Handel hilfreich sein. RFID ist zudem wegen der Datenschutzproblematik gesellschaftlich sehr umstritten. Auch wenn die Proteste abgeebbt sind, sind kritische Organisationen nach wie vor politisch aktiv und begleiten die Einführung der Technologie. Daraus ergibt sich die Notwendigkeit, Umfeld, Stakeholder und Risiken kontinuierlich zu beobachten und sich auf Konflikte bei Gesetzgebung und staatlicher Regulierung der RFIDTechnik einzustellen.

\section{Einführung: Die Eskalation der RFID-Kontroversen}

Auf dem Radarschirm der Politik ist Radiofrequenzidentifikation über Jahrzehnte ein unbedeutender Punkt gewesen, um dann plötzlich zum Auslöser intensiver Debatten zu werden. Das ist für High-Tech-Themen nicht untypisch. Die politische Reichweite von RFID vergrößerte sich gegen Ende der 1990er Jahre erheblich. Ausgelöst wurde dies durch die Verbreitung der Technik in verbrauchernahen Einsatzfeldern und die Entwicklung eines neuen Politikfelds.

Einerseits nahmen Regierungen und Gesetzgeber die Entwicklung der digitalen Zukunft und der »Informationsgesellschaft « strategisch und regulatorisch in den Blick. Andererseits erreichten RFID-Kritiker mit ihren Bedenken nun zahlreiche Bürger. Es kam zu öffentlichen Debatten und medienwirksamen Protesten, die die Politik zu Reaktionen zwangen. Zuerst in den USA, dann auch in Europa wurde RFID Gegenstand parlamentarischer Aktivitäten und Untersuchungen durch Aufsichts- und Regulierungsbehörden. Technikkritiker sowie Daten- und Verbraucherschützer spitzten die Debatten zu und organisierten sogar Boykottbewegungen. Eine wesentliche Rolle spielte dabei das Internet als Kommunikations- und Organisationsmedium für die rasch wachsende Szene vernetzter Initiativen im Feld Datenschutz, Informationsfreiheit, informationeller Selbstbestimmung und Bürgerrechte.

Zu Beginn der 2000er entwickelte sich die politische Debatte für die an der RFID-Einführung in Deutschland und Europa interessierten Unternehmen - insbesondere in Logistik, Handel und IT-Wirtschaft - zum Risiko: Sie beeinflusste die Anstrengungen, 
Standards und ein rechtssicheres Rahmenwerk für RFID auf nationaler wie europäischer Ebene zu schaffen, öffentliche Fördermittel für Forschung und Entwicklung zu gewinnen und schließlich die Akzeptanz beim Verbraucher und Bürger zu sichern.

Der Staat war von Anfang an selbst betroffen. Im Feld der Sicherheitstechnologie ist der Staat ein wichtiger Kunde der IT-Wirtschaft, und er macht Vorgaben für Kunden des gesamten öffentlichen Sektors von Bundeswehr über öffentliche Kreditinstitute und Krankenkassen bis zu - natürlich - Bibliotheken und Archiven.

Eine scharfe, enge, RFID-spezifische Regelung wollten die Unternehmen nicht. Schon gar nicht sollte ein nationaler Alleingang Deutschlands erfolgen: Forderungen etwa nach der Aufnahme von RFDI ins Bundesdatenschutzgesetz und scharfen Strafen gegen Verstöße machten die Industrie nervös. Ein deutsches »Lex RFID« sollte aus Sicht der Industrie verhindert werden. Stattdessen sollte ein für die Unternehmen flexibler Empfehlungsrahmen (»soft law«) auf europäischer Ebene die Konfliktpunkte befrieden. Die deutsche Regierung sollte ihr Gewicht bei der EU für eine sanfte Lösung einsetzen.

Das sollte auch gelingen: Nach ersten Weichenstellungen 2006 (öffentliches Konsultationsverfahren zu RFID) beschloss die Europäische Kommission im Mai 2009 Empfehlungen zu Privatsphäre und Datenschutz bei RFID, die von den EU-Mitgliedstaaten in nationale Maßnahmen umsetzen müssen. Das Ergebnis war also eine flexible Regelung mit Spielraum für Interpretationen. Dabei kam es den RFID-Befürwortern zu Gute, dass die Kommission das »Internet der Dinge « vorrangig als positiven Teil ihrer »Digitalen Agenda«, der Lissabon-Strategie und des 7. EU-Forschungsrahmenprogramms verstanden wissen wollte. Die RFID-Entwicklung sollte zur Wettbewerbsfähigkeit Europas beitragen. Deshalb fielen die EU-Vorstöße zu Datenschutz, Informationspflichten und Sicherheit am Ende sehr maßvoll aus.

Das konnte 2003-2005 aber niemand absehen. Die politische Lage war äußerst problematisch, sowohl in der EU als auch in Deutschland. Mit großem Medienecho starteten Datenschutzorganisationen 2004 eine »Stopp RFID«-Kampagne gegen »Schnüffelchips« und den »gläsernen Verbraucher«. Sie brachten öffentlichen Zorn über die Kundenkarten- und »Future Store«-Projekte des Handelskonzerns Metro. 2005 verursachte der Streit um die Fußball-WM-Tickets politischen Wirbel. Besonders intensiv war die Publizität über RFID zwischen 2004 und 2007, wobei in der Publikumspresse Beiträge zu rechtlichen Aspekten, insbesondere Datenschutz, dominierten - 2004 gar bis zu zwei Dritteln der Presseartikel, wie der Zukunftsreport Ubiquitäres Computing für die Technikfolgenabschätzung des Bundestages feststellte (Deutscher Bundestag, 2010, S. 100). Die Firmen reagierten gereizt. Im August 2005 strahlte das ZDF-Magazin Frontal21 den Beitrag »Der gläserne Bürger - Überwachung per Funkchip« aus, gegen den Metro und der Fußballverband FIFA mit ihren Rechtsanwälten vorgingen. Das ZDF nahm das Video und das Sendemanuskript von der Website, jedoch kursierten beide kurze Zeit später wieder im Internet (LobbyControl, 2005).

Im Bundestag ließ die Opposition aus Grünen, FDP und Linken ab 2004 beim Thema RFID nicht mehr locker: Die Bundesregierung hatte sich mit parlamentarischen 
Anfragen, Entschließungsanträgen, Ausschussberatungen und Plenardebatten zu RFID auseinanderzusetzen. In der 16. Wahlperiode ab 2005 zählt das Dokumentationssystem des Bundestags 102 parlamentarische Drucksachen und 24 Plenarprotokolle zu RFID von der Warenetikettierung bis zum biometrischen Reisepass (Deutscher Bundestag, 2011).

Die von den Bundesministerien für Wirtschaft und Verbraucherschutz ab Sommer 2004 initiierten Konsultationen »RFID und Verbraucherschutz « mit Handel, Industrie, Daten- und Verbraucherschützern hatten einen holprigen Start. Ein von der Standardisierungsorganisation GS1 Germany ausgearbeiteter Entwurf zur Selbstverpflichtung der Wirtschaft stieß auf Ablehnung der an den Konsultationen beteiligten Gruppen wie dem Verein zur Förderung des öffentlichen bewegten und unbewegten Datenverkehrs (FoeBuD) und der Deutschen Vereinigung für Datenschutz (DVD), die den öffentlichen Druck durch ihre Kampagnen ständig erhöhten. Unternehmen sahen sich in der Defensive und genötigt, ihre RFID-Projekte zu rechtfertigen, teilweise zurückzufahren und Daten- und Verbraucherschützern entgegenzukommen (Hamann, 2006).

\section{Das Informationsforum RFID - ein Kind der Berliner Republik}

Die politische Kommunikation der RFID-Befürworter blieb zunächst unterentwickelt, langsam und fragmentiert. Eine Reihe von Spitzen-, Branchen-, Fach- und Berufsverbänden der Wirtschaft setzte sich für RFID ein: der Bundesverband der Deutschen Industrie (BDI), Bitkom für die IT-Wirtschaft, der Elektrotechnik-Verband VDE, der Handelsverband HDE, diverse Logistikverbände, die Gesellschaft für Informatik und andere, unterstützt und ergänzt durch punktuelle Interventionen der Unternehmen mit ihren spezifischen Belangen. Besonders effektiv schien das jedoch nicht.

In die Defensive geraten, warb der Metro-Konzern daher bei vielen Partnern um einen koordinierten, strategischen und themenspezifischen Ansatz. Resultat der Absprachen war im April 2005 die Gründung des Informationsforums RFID e.V. Das IF sollte eine Plattform sein, die den »Gefahren« der »falschen Einschätzungen« und des »großen Informationsdefizits« wehren und »das innovative und wirtschaftliche Potenzial der Technologie« in der öffentlichen Diskussion verdeutlichen sollte, so die Geschäftsführerin Andrea Huber (Stegherr, 2005b, S. 49). Die promovierte Juristin war zuvor Government Affairs Director bei Microsoft Deutschland und Leiterin der Abteilung Internationale Interessenvertretung der Deutschen Telekom. Sie baute das IF auf, dem für fünf Jahre eine Schlüsselrolle im Lobbying und der politischen Öffentlichkeitsarbeit zukommen sollte.

\section{Modell Strategische Allianz}

Das IF war weder Verband noch Ad-hoc-Koalition. Vielmehr handelte es sich um eine Organisationsform neuen Typs: eine Strategische Allianz mit eigener Infrastruktur und professioneller Geschäftsführung, engem Fokus und zeitlich befristetem Auftrag für die politische Kommunikation in Kampagnenform. Das IF wirkte als Projektagentur. Von vornherein war klar, dass das IF nur solange bestehen würde, bis die schwierige politische Lage gemeistert und die Weichen für die allgemeine Gesetzgebung gestellt waren. 
Strategische Allianzen sind Kinder der Berliner Republik: Medienwirksame, schlank strukturierte, projektorientierte, flexible Interessenkoalitionen oft ungewöhnlicher Partner jenseits der etablierten Verbändestrukturen. Die Begrenzung auf ein Ziel oder wenige Themen erleichtert es, Interessengegensätze ungleicher Partner auszuklammern. »Lobby im Schnellboot « und »schnelle Eingreiftruppe der Unternehmen« nannte sie das Fachblatt Politik \& Kommunikation (Stegherr, 2005a, S. 46).

RFID wurde zum Paradebeispiel für das Novum der Strategischen Allianzen. Andere Allianzen der ersten Jahre waren das Informationszentrum Mobilfunk (fokussiert auf gesundheitliche Ängste bei Mobilfunkantennen), die Allianz pro Schiene (die gegen Autobahn- und Flugverkehrausbau Bahninteressen vertritt, und zwar als gemeinsame Plattform von Bahnen, Bahnindustrie, Umwelt- und Fahrgastverbänden sowie Gewerkschaften), die »Existenzfrage Zucker« (mit der sich die Zuckerindustrie zur EU-Zuckerreform zu Wort meldete), die Intiative »Druck gegen Abgaben « (der Druckerhersteller bei der Urheberrechtsnovelle), die »Initiative Luftverkehr« oder »Mehr Bonus für Kunden« (von Handels- und Finanzfirmen gegen das Rabattgesetz). Jüngst kam »Die Lebensmittelwirtschaft« hinzu, eine Allianz von Industrie, Handel, Handwerk und Gewerkschaft NGG, die industriekritischen Vereinen wie Foodwatch die Stirn bieten soll.

Solche Allianzen sind für mehr als einen kurzfristigen Interessenabgleich gedacht, nämlich für eine gemeinsame Steuerung bei komplexen Vorhaben über mehrere Jahre hinweg (von Münchow, 2006, S. 39). Zudem steht die externe Kommunikation, nicht die Gremienarbeit oder Dienstleistungen für Mitglieder im Vordergrund: »Allianzen sind darauf ausgelegt, die Öffentlichkeit und somit die Politik für ihr Thema zu gewinnen. Sie arbeiten nicht ausschließlich im Verborgenen - sie wirken nach außen nicht nach innen. Dies unterscheidet sie grundlegend von einem Verband« (S. 40).

Geschäftsführerin Huber nannte das IF eine »Plattform für Diskussionen über Datenschutz, Verbraucherschutz und andere kritische Themen«. PR- und Politikverantwortliche der Mitglieder trafen sich alle zwei Monate im Arbeitskreis Kommunikation, um sich auszutauschen und zu koordinieren. Das Forum sollte »Scharnierfunktion« haben und Synergien erzeugen:

»Als strategische Allianz spart man Ressourcen und bündelt Know-how, zum Beispiel für Flyer oder die Internetseite. Unternehmen wollen sich zudem gegenüber der öffentlichen Kritik nicht unbedingt einzeln positionen. Sie sind dankbar, wenn eine neutrale Plattform die Diskussion in ihrem Sinne führt. [...] Es gibt bei uns keine hauptamtlichen Funktionäre und keine gewachsene Kassenwartsmentalität, wie sie althergebrachte Verbände zum Teil auszeichnet. Bei uns geht es in erster Reihe ums Thema, für uns zählt der konkrete Erfolg« (Stegherr, 2005b, S. 49).

Die Netzwerkarbeit zwischen Allianz und den Mitgliedern kann als »Lobbying nach innen « bezeichnet werden, so Huber (2007, S. 9). Das Informationsforum RFID folgte auch dem Prinzip, möglichst ungewöhnliche Partner zu einigen. »Je bunter und je breiter eine Allianz aufgestellt ist, desto mehr potenzieller Erfolg steckt in ihr. Gerade diese Breite verleiht der Allianz das Attribut >strategisch» «, betont von Münchow (2006, S. 40). In 
der Tat: Das IF vereinte den IT-Verband Bitkom und die Bundesvereinigung Logistik, Logistiker wie DHL und Intermec, Industriekonzerne wie Volkswagen und Philips, Konsumgüterhersteller wie Procter \& Gamble, Gillette und Henkel, IT-Unternehmen wie IBM, T-Systems, Oracle, Hewlett-Packard, NXP oder SAP und Siemens sowie den Einzelhandelskonzern MetroGroup.

Das war keine allzu große Gruppe. Vielleicht war das gut so, denn das Risiko des Scheiterns ist bei sehr großen Allianzen durch hohen Abstimmungsbedarf, Unterschiede in Struktur und Selbstverständnis der Partner, ungleiche Belastung und Konfliktmanagement ungleich höher, wie Wiebusch (2005, S. 219) anmerkt:

»Dieser Koordinationsbedarf kann eine neue Organisation bei der Umsetzung ihrer Ziele deutlich verlangsamen, möglicherweise sogar lähmen und so die Hoffnungen auf große Schlagkraft enttäuschen.«(S. 219)

Während etwa die Allianz pro Schiene mehr als 50 Mitgliedsorganisationen (darunter viel Nonprofit-Gruppen) umfasst, blieb das IF stets auf rund ein Dutzend beschränkt, fast ausschließlich Großunternehmen. Ob man unter sich bleiben wollte oder die Neurekrutierung nicht gelang, ist unklar. In der Anfangszeit war z. B. mehrfach die Rede davon, die Luftverkehrsbranche einzubeziehen. Das materialisierte sich jedoch nicht. Auffällig ist zudem, dass die IT-Branche breit repräsentiert war, der Einzelhandel aber nicht. Weder war der Dachverband HDE Mitglied noch die großen deutschen Handelsgruppen wie Rewe, Edeka, Tengelmann, Otto, Arcandor oder die Discounter Aldi, Lidl, Netto oder Schlecker. Das IF suchte allerdings oftmals punktuell nach Partnern außerhalb seiner Mitgliedschaft: So wurden vom IF diverse Positionspapiere in die Politik eingespeist, die die Logos des Einzelhandelskonzerns GroupeCarrefour, des HDE, BDI, des Deutschen Industrie- und Handelskammertags (DIHK), des Automobilindustrieverbands VDA oder des Markenverbands trugen (Informationsforum RFID, 2011).

Bezeichnenderweise stellten die Mitglieder keinen Unternehmenslenker als Vorsitzenden an die Spitze des IF-Trägervereins, sondern den Geschäftsführer des Fraunhofer-Instituts für Materialfluss und Logistik und Professor der TU Dortmund, Michael ten Hompel. Außer ihm war allerdings am IF keine einzige akademische Einrichtung beteiligt. Mit dem Wissenschaftler als Frontmann und Sprecher wurde eine neutrale Expertenautorität des IF suggeriert.

\section{Lobbying und begleitende öffentliche Kommunikation}

Die Lobbyarbeit des IF umfasste das Kontaktmanagement mit Entscheidungsträgern, für die Hintergrundgespräche, Parlamentarische Abende, Fachveranstaltungen in Bund und Ländern und sonstige Events arrangiert wurden. Das IF trat mit seinen aus den Mitgliedsunternehmen rekrutierten Experten bei Ausschussanhörungen und bei Konsultationen auf, steuerte Positionspapiere, Rechts- und Fachgutachten sowie Studien und Umfragen bei. Das IF war nicht nur in Deutschland aktiv, sondern auch in Brüssel, wo es mit Schwesterorganisationen wie dem britischen RFID Centre oder der RFID Platform Nederland zusammenarbeitete. Der Öffentlichkeitsarbeit dienten mehrere Websites 
für Politik, Journalisten, Wissenschaftler und Bürger. Das IF produzierte Broschüren, Berichte, Materialien für die Verbraucherkommunikation. Es organisierte Interviews, Pressegespräche sowie Messeauftritte (z. B. CeBIT) und Roadshows für mittelständische Unternehmen. Zum Teil ließ sich das personell schlanke IF (drei feste Mitarbeiter) von Public-Affairs-Agenturen wie Pleon und externen Pressebüros sowie den Stäben der Mitgliedsfirmen unterstützen.

Der erste Aufschlag fand an prominenter Adresse statt: im Leibniz-Saal der Berlin-Brandenburgischen Akademie der Wissenschaften, wo sich das IF Abgeordneten, Ministerialbeamten und Journalisten vorstellte. Der Auftakt erfolgte spät, erst ein halbes Jahr nach Eröffnung des IF-Büros. Das Timing geriet unter die Räder der großen Politik: »Ursprünglich wollten wir im Herbst 2005 richtig durchstarten. Das hat sich leider verzögert, weil sich die Koalitionsverhandlungen zwischen CDU und SPD sehr lange hingezogen haben «, berichtete Geschäftsführerin Huber später. »Es standen zu dieser Zeit einfach keine Sprecher aus der Politik zur Verfügung, sodass wir unsere Auftaktveranstaltung auf Januar 2006 verschieben mussten « (Informationsforum RFID, 2009d). Kritiker wurden dazu nicht eingeladen. Sie kamen trotzdem. »Mitglieder des FoeBuD standen im Januar 2006 tatsächlich mit Plakaten vor der Location unserer Auftaktveranstaltung «, wunderte sich Huber noch drei Jahre später (Informationsforum RFID, 2009d, S. 8).

Per Pressemitteilung attackierten FoeBuD und DVD »die unkonstruktive Haltung der RFID-Industrie, die lieber kritikfreie Jubelveranstaltungen durchführt statt sich konstruktiv am kritischen Dialog zu beteiligen.« Kritik solle mit einer PR-Offensive erstickt werden, während die Wirtschaft bei den Konsultationen der Bundesregierung »Hinhaltetaktik« betreibe. Sie wolle »nur Zeit gewinnen, um Fakten zu schaffen« (FoeBuD, 2006).

Die Kritiker lagen durchaus richtig. Zu jener Zeit waren viele RFID-Projekte noch weit von Serienreife und Marktdurchbruch entfernt. Die Arbeit des IF sollte eine zu frühe Verrechtlichung der Technologie verhindern. Dialogverweigerung kann man der IF nicht grundsätzlich vorwerfen. Allerdings suchte sich das IF sehr genau aus, mit wem es den öffentlichen Dialog führte. So nahmen IF-Vertreter eher auf Podien mit staatlichen Datenschutzbeauftragten oder Repräsentanten der Verbraucherzentralen Platz als mit FoeBuD und DVD. Das IF beobachte ihre Aktivitäten und nahm ihre Argumente auf. Zu keiner Zeit ließ sich das IF aber auf Schaukämpfe mit ihnen ein, deren Polarisierungsversuche zunehmend ins Leere liefen.

\section{Einflussnahme durch Politikberatung und Kooperationsprojekte}

Vorrang hatten die tatsächlichen Entscheidungsträger. Für sie wollte das IF konstruktiver Politikberater mit Expertenstatus und Partner sein, keine aggressiv fordernde, kritisierende oder jammernde Interessenvertretung. In zahlreichen Publikationen fällt auf, wie häufig das IF und das bei RFID federführende Bundesministerium für Wirtschaft und Technologie sich gegenseitige Wertschätzung ausdrückten. Der gute Zugang zu Fachreferaten und zur Hausleitung ermöglichte gemeinsame Projekte und Veranstaltungen. Als Deutschland 2007 die EU-Ratspräsidentschaft innehatte, gewährte das Ministerium bei der EU-High-Level-Konferenz »RFID: Towards the Internet of Things« dem IF eine 
prominente Expertenrolle, die sich auch im Abschlussbericht »European Policy Outlook RFID « niederschlug - ein Arbeitspapier mit direktem Einfluss auf Brüssel (Bundesministerium für Wirtschaft und Technologie, 2007). Das Ministerium förderte eine von IF und dem Softwarehaus Oracle betreute bundesweite Veranstaltungsreihe für den Mittelstand (Informationsforum RFID, 2008a). Beide schrieben gemeinsam einen (vom IF verwalteten) RFID-Mittelstandspreis aus (Informationsforum RFID, 2009). Das Ministerium war Schirmherrn für den IF-Logo-Wettbewerb »RFID zeigt Gesicht!«, bei dem Designstudenten Entwürfe für eine Kennzeichnung von verbrauchernahen Anwendungen einreichten (Informationsforum RFID, 2008b) - eine Aktion, die der FoeBuD übrigens mit einem Gegenwettbewerb für ein Warnzeichen beantwortete (FoeBuD, 2008).

Ein kurzer Draht bestand auch zur EU-Kommission, die das IF in seine StakeholderBeratungsgruppe zu RFID einlud. Sie rief zur CeBIT 2009 eine auf drei Jahre angelegte Initiative »RFID in Europe« ins Leben, in die das IF als eine von 25 RFID-Institutionen aus 17 Staaten eintrat. Das IF wurde zum Gründungsmitglied des EU-finanzierten RACEnetworkRFID (»Raising Awareness and Competitiveness in Europe«). Das IF übernahm ganz offiziell für die EU die Kommunikationsaufgabe, »Public Awareness « für RFID zu fördern, was das IF als Förderung der »Akzeptanz« übersetzte (Quack, 2009).

Bei so enger Kooperation ist nicht überraschend, dass jegliche öffentliche Kritik des IF an der Politik diplomatisch verpackt wurde. In Interviews und Pressemitteilungen verzichtete das IF auf harsche Wortwahl. So war es etwa bei der Branchenkritik an der Empfehlung der Kommission, Verbrauchern in jedem Fall eine Chip-Deaktivierung zu ermöglichen. Der Mitgliedsverband Bitkom äußerte sich oft prononcierter: Bei der CeBIT 2009 betonte das IF die positive Zusammenarbeit mit Brüssel, während Bitkom die Empfehlung als überflüssig bezeichnete und vor bürokratischen Hemmnissen warnte, die den Markt verunsicherten und Investitionen behinderten (Kümmerlen, 2009). So teilten sich die Akteure die Rollen.

Selbstverständlich lobbyierten und informierten die IF-Mitgliedsverbände und Mitgliedsunternehmen parallel eigenständig in Sachen RFID weiter, oftmals von Beratern, Agenturen und Anwaltskanzleien unterstützt. Die dabei eingesetzten Ressourcen sind nur punktuell bekannt geworden, beispielsweise im Sponsoring: Das Bundesministerium für Wirtschaft und Technologie ließ sich 2007 die EU-High-Level-Konferenz »RFID: Towards the Internet of Things « u.a. von Metro (30.000 Euro), IBM (30.000 Euro), dem mit IF en kooperierenden IT-Verband AIM (10.000 Euro) und dem Softwarekonzern SAP (15.000 Euro) sponsern (Bundesministerium des Innern, 2009). Die Agentur Pleon, die sowohl für das IF als auch die Metro Group arbeitete, trat mit 30.000 Euro als Kofinanzier eines Seminars für Lokaljournalisten der Bundeszentrale für politische Bildung auf, Thema »Intelligenz im Supermarkt - mit dem Einkaufswagen in die Zukunft« (Denkler, 2007).

\section{Politische Bilanz und vorläufiges Ende}

Trotz vieler Anfeindungen hat das IF fraglos viele Ziele erreichen können. Dazu gehört die Beruhigung und Normalisierung der öffentlichen Debatte, aber explizit auch, was 
regulatorisch nicht geschah: Kein deutsches Gesetz wurde auf den Weg gebracht, keines geändert. Die Bundesregierung entschied, es gebe keinen Bedarf (Deutscher Bundestag, 2008).

Auch auf EU-Ebene kam es zu keinem Rechtsakt. Statt Parlament und Rat eine Richtlinie mit Gesetzeskraft vorzuschlagen, beließ es die Kommission im Mai 2009 bei einer Empfehlung für die Mitgliedstaaten (Europäische Kommission, 2009a). Das wurde im »Aktionsplan für Europa « zum »Internet der Dinge« vom Juni 2009 bestärkt (Europäische Kommission, 2009b). Das Europäische Parlament hingegen forderte im Juni 2010 konsequentere Datenschutzdurchsetzung und gab der Kommission den Auftrag, einen klaren Rechtsrahmen auszuarbeiten. Es sei absehbar, dass die von der Kommission bevorzugte Selbstregulierung der Wirtschaft nicht die gewünschten Ergebnisse bringen werde (Europäisches Parlament, 2010). Es ist offen, ob und wie die Kommission dem künftig folgt. Sie wartet erst einmal die nationalen Umsetzungsberichte ab. Die EU-Empfehlungen sind nach heutigem Stand sehr liberal und kein regulatorisches Risiko. Das mag sich künftig ändern, doch bis dahin dürfte die RFID-Technologie fest im Alltag verankert sein.

In der Gesamtsicht dürften die Träger des IF mit der fünfjährigen Leistung zufrieden sein. Festzuhalten ist, dass das Erfolgsgeheimnis nicht allein beim kleinen IF-Büro lag, sondern im Multi-Voice-Lobbying, bei dem die Mitglieder ihre jeweiligen Kanäle selbstständig nutzten. Das IF war eher Clearingstelle als Kommandozentrale.

2010 endete die Tätigkeit des IF in Berlin. Die Berliner Geschäftsstelle und der Trägerverein wurden aufgelöst, Huber wechselte zum Verband Deutscher Kabelnetzbetreiber, das IF als »Marke« kam unter das Dach von GS1 Germany in Köln - weitab der politischen Schaltstellen. In Köln geht es nun um technische Details und Beratung der RFID-Anwender bei der Umsetzung der EU-Empfehlungen. Schon im IF-Jahresbericht 2009 deutete Huber an:

»Die beste Entwicklung - auch für unsere Mitglieder - wäre eigentlich, dass man uns gar nicht mehr braucht. Wenn wir irgendwann alle kritischen Fragen lösen können und RFID in der Politik und bei den Verbrauchern akzeptiert ist, hat sich unser Zweck erfüllt« (Informationsforum RFID, 2009c, S. 11).

Die tatsächliche Auflösung anderthalb Jahre später mag man als Indiz deuten, dass der Auftrag erfüllt war. Ganz verschwinden sollte das IF nicht. »Die Markenbekanntheit hat für uns eine große Bedeutung. Daher wird das RFID-Informationsforum nach außen hin seine Visibilität weiterhin behalten, nur organisatorisch sind die Stakeholder breiter aufgestellt«, erläuterte der GS1-Geschäftsführer Jörg Pretzel im Fachmagazin RFID im Blick. GS1 habe eine »viel breitere Unternehmens- und Branchenbasis«. Der neue Schwerpunkt: »Wir wollen das Thema Verbraucher- und Datenschutz enger an die operativen Umsetzungsthemen heranführen, wobei wir dieses auf einer größeren Basis behandeln « (RFID im Blick, 2011). 


\section{Lobby-Lektionen}

Was lässt sich aus dem Interessenvertretungsmodell des Informationsforums RFID lernen, insbesondere für Bibliotheken? Zum einen fällt die konsequent kooperativ-konstruktive Haltung auf, die in der seinerzeit äußerst kritischen Lage ja nicht selbstverständlich war. Das IF mied jeglichen polarisierenden Streit, jeden scharfen Angriff, aber auch jedes Jammern wie der Teufel das Weihwasser. Das erfordert hohe Kommunikationsdisziplin und ist mehr als nur eine Stilfrage. Das IF blieb stets bei seinen Kernbotschaften, was durch die zwar nicht homogene, aber recht kleine Mitgliedergruppe erleichtert wurde.

Klare Adressatenorientierung durchzog sämtliche Aktivitäten. Für alle Zielgruppen (Politiker, Beamte, Journalisten, Verbraucher) gab es geeignete Dialogangebote. Das IF suchte die Kongruenz mit der Themen-Agenda der Politik. Es bot sich als Partner, Berater und Dienstleister an, um die Ziele der Politiker und Behörden erreichen zu helfen und diese in einem guten Licht dastehen zu lassen. Das IF beanspruchte Meinungsführerschaft beim RFID-Thema. Dennoch ließ es sich gern in die Aktivitäten politischer Partner einbinden und band sie ein. Das IF setzte sogar im offiziellen Auftrag und mit finanzieller Förderung des Wirtschaftsministeriums und der EU-Kommission Kommunikationsprojekte um. Es wurde zum ausführenden Organ der Politik.

Die ständige Suche nach Projekt- und Kooperationspartnern sowie Vernetzung und behutsamer Koordination war ein erkennbares Markenzeichen des IF. Dieser OutreachAnsatz vergrößerte die Reichweite und signalisierte der Politik, dass das IF zahlreiche informell Verbündete hatte. Das IF vertrat Konzern- und Brancheninteressen, aber argumentierte sektorübergreifend mit dem Nutzen für andere, für Mittelstand, Verbraucher, Gemeinwesen, Wirtschafts- und Technologiestandort.

Ein Mehrwert ergab sich auch aus der internationalen Orientierung: Das IF vermittelte zwischen Berlin und Brüssel sowie zwischen EU-Mitgliedsländern. Vernetzt mit ähnlichen RFID-Plattformen in Europa, beteiligte es sich an der europäischen Debatte und war quasi die offizielle Vertretung und Stimme der deutschen RFID-Anwender. Diese Stellung im Ausland hatte Rückwirkung auf den Einfluss im Inland.

Der Großteil der Arbeit kann transparent genannt werden: Wer hinter dem IF stand und welche Interessen verfolgt wurden, war kein Geheimnis. Als fragwürdig erscheint höchstens die Berufung eines Wissenschaftlers als Vorsitzenden eines Vereins, der mit Wissenschaft wenig zu tun hatte. Substanziell konnten allerdings auch hyperkritische Watchdog-Gruppen wie z. B. LobbyControl dem IF keine Vorwürfe über unsaubere Arbeit machen.

\section{RFID-Lobbying bei Bibliotheken}

Verglichen mit den Ressourcen internationaler Konzerne muss das RFID-Lobbying bei Bibliotheken kleine Brötchen backen, keine Frage. Auch strukturell und inhaltlich sind Unterschiede festzustellen: Beim Fokus und den Zielen, bei den politischen 
Entscheidern, den Argumenten und den Instrumenten. Das IF-Modell ist keine perfekte Blaupause. So wird Bibliotheken eher als den IF-Mitgliedern Legitimität als Träger öffentlicher Interessen zugestanden, was Möglichkeiten einer härteren kommunikativen Gangart einschließt. Bibliotheken haben durch ihre Nutzer eine viel breitere Basis, die sich mobilisieren ließe. Das IF versuchte das Vertrauen der Verbraucher durch Informationskampagnen zu gewinnen, für RFID mobilisieren wollte sie sie nicht. Bibliotheken könnten hier theoretisch anders vorgehen - wer Bürger für die auskömmliche Finanzierung der Bibliotheken mobilisiert, könnte auch die Modernisierung der Technik einschließen.

Andererseits ist das IF ein Vorbild, wie sich eine Interessengruppe ein Profil als gefragte Experten geben kann, die die Agenda der Politik unterstützen, statt sie zu kritisieren. Anpassung der Strategie an Bedürfnisse der Entscheidungsträger, Lobbying als Politikberatung, Einflussnahme durch Kooperationsprojekte, Vernetzung und Dienstleistung sind hier die Stichworte.

\section{Lobbying unter Haushaltszwang}

Zwar zielte die Wirtschaft auch auf staatliche Subventionen für die RFID-Forschung und Verbreitung ab, doch stand bei ihrer Lobbyarbeit der rechtliche Rahmen im Vordergrund. Bibliotheken haben dagegen bei ihrer Beschaffung vorrangig ein finanzielles Problem. Sie müssen die öffentlichen Haushälter in Ländern und Kommunen überzeugen. Das ist ein völlig anderer Adressatenkreis. Nur wenige dieser Politiker verfolgen intensiv und fachkundig die rasante Entwicklung der Informationstechnik oder speziell der Bibliotheksinfrastruktur im digitalen Zeitalter. Pauschal gesagt, sind Politiker nicht in der Lage, von sich aus Aufwendungen für die technische Ausstattung von Bibliotheken zu begründen und zu rechtfertigen. Das steht bisweilen in umgekehrt proportionalem Verhältnis zur Selbsteinschätzung. Andere Interessengruppen haben es zudem von vornherein leichter, freundliche Akzeptanz für Investitionen komplexe Technik zu finden: wenn Meldeämter leistungsfähige Computer brauchen, Forscher und Gesundheitseinrichtungen Laborgeräte oder Feuerwehren Einsatzsimulatoren und Spezialgerät.

Investitionen in RFID kosten viele Steuermittel. An RFID in Bibliotheken ist aber per se politisch nichts Interessantes, es ist ein Spezialwunsch unter vielen, mit dessen Erfüllung Politiker nicht zum Helden der Nachbarschaft werden. Politiker haben in Zeiten klammer Kassen ohnehin Verteilungskonflikte zu bewältigen, die für die Wiederwahl kritisch sein können. So dürften sich die skeptische Argumente zugespitzt so zusammenfassen lassen: »Das ist purer Luxus«, »Für das Geld könnte man auch viele Bücher kaufen«, »Das versteht bei mir im Wahlkreis keiner«, »Meine Kinder wollen auch immer neues Computerspielzeug «, »Wenn wir das bewilligen, müssen wir beim Hallenbad sparen« oder, ganz egoistisch, »Bis sich das rentiert, bin ich in Rente.«

Ohnehin sind Bibliotheken stark von der Haushaltskonsolidierung betroffen. Viele stehen unter Haushaltssperren, von ihnen werden Etatkürzungen oder Gebührenerhöhungen verlangt. Erwerbungsetats sinken, Öffnungszeiten und Veranstaltungen werden reduziert und Bauprojekte gestrichen, Personalstellen bleiben unbesetzt oder geraten 
unter den Rotstift, und manchmal droht die Schließung. Von einer »Aushöhlung « dieser Bildungsinfrastruktur zu sprechen, ist sicher nicht übertrieben (Deutscher Bibliotheksverband, 2010).

Das ist also kein freundliches Umfeld, um für teure Technik-Investitionen nebst hohen Folgekosten zu werben. Die allgemeine Situation ist nicht neu, weshalb Bibliotheken und ihre Verbände sich immer stärker der Lobby- und Medienarbeit zuwenden. Sie suchen international nach Vorbildern und tauschen sich über Best-Practice-Beispiele aus, wie auch an einer wachsenden Zahl von Lobby-Handreichungen der Verbände sowie Ratgebern und Handbüchern speziell für Bibliotheken abzulesen ist. Vorerst bleibt die Beobachtung richtig, dass Bibliotheken selbst in bildungs- und innovationspolitischen Debatten Zaungäste sind, nur punktuell PR-Erfolge verzeichnen, Defizite bei der Vernetzung aufweisen und strategisch, taktisch und operativ »nur ansatzweise das Niveau einer Pressure-Group« erreichen (Ratzek, 2010, S. 33). Andere Interessengruppen kontern das Politiker-Argument, die Kassen seien leer, mit höherem politischem Druck, auf den die Politiker dann reagieren müssen. Das fällt Bibliotheken schwer.

\section{Vier Argumentationslinien für die RFID-Beschaffung}

Druck auszuüben, ist allerdings nicht der einzige Weg. Es geht durchaus um inhaltliche Argumente: solche, die den Nutzen für den Politiker und seine Agenda sowie nicht bibliotheks-, kultur- und bildungsspezifische Ziele betonen. Wie im Lobbymodell des IF geht es um die Kongruenz eigener und Politikerinteressen. Statt über eigene Probleme zu sprechen, spricht man über die Gemeinsamkeiten der Agenda - und präsentiert Lösungen.

Hier lohnt sich der Blick auf internationale Erfahrungen. Der US-Bibliothekenverband ALA beispielsweise hat in seiner »Advocacy University « ein umfassendes Argumentationspaket geschnürt, wie man auch in Zeiten eine Wirtschaftskrise Wert und Rendite von Bibliotheksinvestitionen gegenüber Politik und Medien vermittelt: Das »Advocating in a Tough Economy Toolkit« ist ein Werkzeugkasten mit Sprechzetteln, Fallbeispielen, Checklisten und Hinweisen für den Dialog mit Politikern, Behördenchefs und Journalisten. Auch wie man Bürger mobilisiert und mit anderen Organisationen Bündnisse aufbauen kann, wird erläutert (American Library Association, 2011).

Darauf aufbauend könnte man die zentralen politischen Argumente für RFID-Beschaffungsinvestitionen in vier Komplexe zusammenfassen: das Kundenservice-Argument, das fiskalische Argument, das wirtschaftspolitische Argument und das High-Tech-Argument.

\section{Das Kundenservice-Argument}

RFID ist ein Beitrag zu höherer Leistungsfähigkeit im Dienst am Kunden - durch Schnelligkeit, Bedienungsfreundlichkeit und Reduzierung von Fehlern. Dass Verwaltungen und öffentliche Betriebe bürgernah, im Sinne von Dienstleistung kundennah und nutzerorientiert sein sollen, hat hohe Priorität bei Politikern und Verwaltungsleitern. Die 
Ansprüche der Bürger sind gewachsen. Das seit den 1990ern populäre »Neue Steuerungsmodell« (NSM oder New Public Management) kennt Leistungskennziffern, Benchmarkings, Produktkataloge, Outputs, Qualitätsmanagement. Auch wenn der Stern des NSM gesunken ist: Bürgermeister, Landräte, Minister sind stolz, wenn sie die unternehmerische Leistungsfähigkeit ihrer Einrichtungen bilanzieren können.

\section{Das fiskalische Argument}

RFID spart bares Geld - die Wirtschaft setzt auf RFID und weiß, was sie tut, wenn sie hier massiv investiert. Sie will Kosten sparen, Abläufe optimieren, die Wirtschaftlichkeit, Leistungs- und Wettbewerbsfähigkeit verbessern. Es geht um Rationalisierungseffekte.

Was für die Wirtschaft richtig ist, kann für Bibliotheken und öffentliche Haushalte nicht falsch sein: RFID liefert höhere Effizienz der Dienstleistungen und eine bezifferbare Rendite der durch Investitionen verbesserten unternehmerischen Tätigkeit.

Nun sind Politiker gegenüber den Amortisierungsversprechen bei IT-Investitionen inzwischen skeptisch geworden. Aus gutem Grund und schlechten Erfahrungen heraus: Hardund Softwareanschaffungen werden ständig von der technischen Entwicklung überholt, was bei den komplexen IT-Systemen dezentralisierter öffentlicher Verwaltungen besonders problematisch ist und teuer wird. Das ist auch bei Bibliotheks-IT nicht abzustreiten. Gleichwohl können Bibliotheken bei RFID auf eine lange Nutzungsdauer verweisen, und ihre Systeme sind kleiner als in der allgemeinen Verwaltung.

Es wäre aber falsch, das fiskalische Argument nur auf den IT-Betrieb zu beziehen. Vielmehr geht es um den fiskalischen Wert der Bibliotheken für die Ziele, die Staat und Kommunen in Wirtschafts-, Arbeitsmarkt-, Sozial-, Bildungs- und Standortpolitik mit anderen Haushaltsausgaben erreichen wollen. Bibliotheken sind wirtschaftliche, effektive Dienste, die diese Ziele erreichen helfen. Eine Investition in RFID erhöht diesen Beitrag zur besseren Ausnutzung begrenzter Haushaltsmittel. Auch das kann man vorrechnen.

Interessant ist RFID auch deshalb, weil dafür z.T. Fördermittel der EU bereitstehen. Zwar ist eine Kofinanzierung mit eigenen Haushaltsmitteln notwendig, die Hebelwirkung ist jedoch dank der EU-Gelder ungleich größer.

Ein weiteres fiskalisches Argument ist das, welches Personalvertretungen und Gewerkschaften naturgemäß nicht gerne hören, Haushaltspolitiker aber schon: RFID hat Effekte auf die Personalbewirtschaftung. Einsparung und Umwidmung von Stellen und Aufgaben ist möglich. RFID ist pauschal kein Arbeitsplatzvernichter, aber sehr wohl ein Rationalisierungsinstrument. RIFD sichert qualifizierte Arbeitsplätze, macht aber Routineaufgaben entbehrlich. Eine Bibliothek, die Personaleinsparungen erbringen muss, hat hier ein gutes Argument in der Tasche: Wenn wir mit weniger Mitarbeitern mehr leisten müssen, dann bitte mit einer Technik, die uns das ermöglicht. 


\section{Das wirtschaftspolitische Argument}

RFID heißt Wirtschaftsförderung. Die Anwendung und Beschaffung von RFID kann die regionale Wirtschaft stärken. Projekte der öffentlichen Hand zur Einführung neuer Technologien demonstrieren die Marktfähigkeit von Anwendungen und lösen so private Investitionen aus. Bibliotheken haben eine sichtbare Vorbild- und Vorreiterfunktion. Die öffentlichen Hände haben durch ihre Beschaffungs- und Dienstleistungsaufträge bei der RFID-Einführung und Systemwartung zudem einen direkten Effekt auf die Auftragsund Ertragslage sowie die Wettbewerbsfähigkeit privater Unternehmen. Haushaltsmittel für RFID-Investitionen fließen in die Kassen des örtlichen Mittelstands, der sich in der Anwendung wertvolle Erfahrungen und Know-how für den Markt sichert, auf dem RFID eine wachsende Rolle spielt - in Handel, Logistik und Industrie.

Weiter vergrößern Bibliotheken allgemein das Humankapital, indem sie Arbeitnehmern und Arbeitssuchenden sowie Menschen in Ausbildung helfen, ihre Beschäftigungsfähigkeit zu sichern und auszubauen. Durch RFID verbesserte Bibliotheksdienstleistungen vergrößern diese Möglichkeiten.

Diese Multiplikatoreffekte sollte man nicht unerwähnt lassen. Ganz besonders, wenn die Region sich einen Wachstums- und Innovationsplan gegeben hat oder sich an einem Wirtschaftsfördercluster beteiligt.

\section{Das High-Tech-Argument}

Ans wirtschaftspolitische Argument schließt sich das technologiepolitische an: Öffentliche Pilotprojekte haben Signal- und manchmal Leuchtturmwirkung für Technologien. RFID-Ausrüstung ist eine strategische Frage des Technologiestandorts, die der Träger der Bibliothek nicht unbeantwortet lassen kann. Nur Bibliotheken mit ausgebauten digitalen Dienstleistungen haben eine Zukunft. RFID ist unverzichtbares Element dieser Infrastruktur. Einrichtungen ohne RFID würden den Anschluss ans moderne Bibliothekswesen verlieren, sich abkoppeln und isolieren. Mit Luxusspielzeug hat das nichts zu tun: Ein zentraler Informationsdienstleister des 21. Jahrhunderts kann nicht mit den Technologien des 20. Jahrhunderts arbeiten. So wie der Nutzer heute in Bibliotheken Datenbanken und digitale Medien findet und nicht nur Bücher, so wie er elektronische und keine Zettelkataloge verwendet, so ist das Herz einer Bibliothek heute die IT im Hintergrund.

Überdies bietet die RFDI-Einführung der Bibliothek interessante Projekte von hoher Komplexität für die angewandte Forschung - ein Thema für die örtliche Universität, Fachhochschule oder Technikinstitute, die damit in der Wissenschaft oder einem Forschungscluster punkten können.

Diese politischen Argumente grenzen sich von den klassischen Argumentationslinien der Bibliotheksfinanzierung ab: Hier ist nicht oder nur indirekt die Rede von Kulturgütern, allgemeiner Bildungsbeteiligung und Chancengleichheit, dem Recht auf Informationszugang, sozialer Inklusion, von der Rolle als Begegnungs- und Kommunikationsstätte oder pädagogischen Aufgaben. So wichtig diese Argumente allgemein auch sind: Gerade 
bei Technikinvestitionen wie RFID gerät man mit »weichen « Begründungen in eine rhetorische Sackgasse. RFID-Chips erfüllen keine kulturellen, sozialen oder pädagogischen Funktionen. Sie ermöglichen es höchstens durch Effizienzgewinne, dass sich Mitarbeiter mehr auf diese konzentrieren können.

\section{Themenmanagement und kritisches gesellschaftliches Umfeld}

Instruktiv ist die Arbeit des Informationsforums RFID für den sensiblen Umgang mit den issues, jenen kritischen, konflikthaften Themenfeldern, die externe Akteure - Anspruchsgruppen oder Stakeholder genannt - mitgestalten und bewusst eskalieren können.

Das strategische Kernproblem liegt darin, die Konfliktfelder und die einflussreichen Akteure rechtzeitig zu erkennen. Das ist die Aufgabe eines Frühwarnsystems oder der strategischen Frühaufklärung. Damit beschäftigt sich seit den 1970ern die Disziplin des Issues Management, ein Teilgebiet der Strategischen Managementlehre und der Risikokommunikation. Auf kontinuierliche Umfeld-Beobachtung soll danach viel Wert gelegt werden.

Zentral ist dabei die Problematik der »schwachen Signale«. Signale für Werte- und Erwartungswandel bei Stakeholdern und künftige Konflikte sind zu Anfang so schwach, dass sie oft übersehen werden. Erkennt man sie aber, hat man eine Chance, mit großem Spielraum frühzeitig in den Thematisierungsprozess einzugreifen, solange Deutungsmuster in Politik und Öffentlichkeit noch nicht festgelegt sind. Das Fenster schließt sich und schränkt die Handlungsmöglichkeiten ein, je mehr Akteure und Medien in die Thematisierung einsteigen (Rawe \& Schulz, 2005, S. 13).

Handel und Industrie übersahen die »schwachen Signale« und gerieten in die Defensive. Bibliotheken sollten den Fehler nicht wiederholen. Konkret geht es um das Vertrauen in die RFID-Technologie und ihre Akzeptanz. Dieselben Akteure, die Handel und Industrie relevant waren, sind grundsätzlich auch Stakeholder RFID-nutzender Bibliotheken. Dieselben Befürchtungen und Zweifel, die die Einstellung zu RFID in Wirtschaft und Behörden prägen, sind für Bibliotheken relevant - auch wenn der spezifische Zuschnitt anders ist.

Bibliotheken sind gut beraten, dieses Umfeld mit seinen issues und Akteuren zu beobachten und zu analysieren. Immerhin ist der RFID-Einsatz in Bibliotheken mit den Funktionen für kommerzielle Lagerverwaltung und Inventur, im kundennahen Bereich aber auch mit Abrechnung, Kundenkarten und Diebstahlprävention der Einzelhandelsunternehmen vergleichbar - also genau jenen Einsatzgebieten, die für politische Kontroversen gesorgt haben. Ob und wie Konfliktthemen aktiv gesteuert werden müssen oder ein Dialog mit den Akteuren begonnen werden muss, ist eine Einzelfall-Entscheidung. Immun gegen Konflikte sind Bibliotheken jedenfalls nicht, so sehr sie auch beteuern, ein datensparsames, sicheres RFID-Modell zu betreiben. 


\section{Thematischer Kontext der RFID-Problematik}

Beim Einzelhandel lag das Datenschutzproblem auf der Hand, weniger wegen der Warenlogistik als vielmehr wegen der marketingrelevanten Verknüpfung mit Kundendaten über Einkaufsverhalten und persönliche Profile. Hier haben die Befürchtungen der Bürger, trotz mancher Übertreibungen, durchaus eine reale Basis. Ebenso plausibel sind die Probleme in der Sicherheitstechnik und Überwachung.

RFID-Befürworter kämpften mit dem Gespenst der »totalen Kontrolle«, das im vergangenen Jahrzehnt in Deutschland Zehntausende zu Protestaktionen und Demonstrationen (»Freiheit statt Angst«) auf die Straße brachte. Diese Mobilisierung belegt die Fähigkeiten von Interessengruppen wie FoeBuD oder Deutscher Vereinigung für Datenschutz (DVD), die seit Jahren Begriffe wie »Schnüffelchips« prägen. Sie arbeiten mit einer Koalition anderer Gruppen zusammen, so dem Chaos Computer Club (CCC), dem Forum InformatikerInnen für Frieden und gesellschaftliche Verantwortung (FIfF), dem Förderverein Informationstechnik und Gesellschaft (Fitug), der Humanistischen Union und der Internationalen Liga für Menschenrechte.

Das Spektrum reicht also von Netzaktivisten bis zu Bürgerrechtlern. Sie besetzen Nischen im Verbändewesen und sind keine Massenorganisationen. Gleichwohl ist ihr Einfluss nicht zu unterschätzen. So hat der Deutsche Bundestag den FoeBuD-Vorsitzenden und die Sprecherin des CCC 2010 als Sachverständige in die Enquete-Kommission Internet und digitale Gesellschaft (die auch eine Projektgruppe Datenschutz und Persönlichkeitsschutz umfasst) berufen, wo sie gleichberechtigt mit Bundestagsabgeordneten und anderen Sachverständigen, etwa ranghohen Wirtschaftsvertretern und Professoren, arbeiten. In der Enquete, die für eine Wahlperiode arbeitet, sind als Sachverständige weitere Vertreter von Organisationen zu finden, die bei der RFID-Debatte eine relevante Rolle gespielt haben, so die Gewerkschaft Ver.di oder der Verbraucherzentrale Bundesverband.

Wesentlich für den Einfluss von Nichtregierungsorganisationen ist es, die öffentliche Agenda über die Medien beeinflussen zu können. Ein Beispiel für Medienerfolge sind die »Big Brother Awards« des FoeBuD und seiner Bündnispartner, die sie als »Oscars für die Datenkraken « bezeichnen. Sie gingen mehrfach an RFID-Anwender: an den MetroKonzern für seinen »Future Store« (2003), an den Deutschen Fußball-Bund wegen der WM-Tickets (2006), an die Deutsche Bahn wegen der BahnCard 100 (2008), an die Modemarke Peuterey und ihren Vertriebspartner Torsten Müller (2011), weil sie Kleidung mit verdeckt integriertem RFID-Chip in Verkehr brächten, der berührungslos auslesbar sei, ohne dass die Kunden das bemerkten (FoeBuD, 2011). FoeBuD \& Co suchen weiter nach aktuellen Fällen. Es ist nicht ausgeschlossen, dass ein »Big Brother Award « demnächst Bibliotheken trifft - was sicher ein großes unangenehmes Medienecho mit sich brächte.

Das Nischenthema RFID erhält trotz seiner technischen Komplexität Aufmerksamkeit durch einen sensiblen rechtspolitischen Kontext und mediale Symbolik. Datenschutz und informationelle Bürgerrechte haben breite öffentliche Debatten ausgelöst, von Google Street View bis zu Datenlecks bei Facebook oder dem Apple iPhone, von der Mitarbeiterüberwachung bei Lidl, Deutscher Bahn und Deutscher Telekom bis zur 
EasyCash-Affäre bei EC-Karten, von der Arbeitnehmerdatenbank Elena bis zur elektronischen Krankenakte und zu biometrischen Ausweisen. Dazu gehören ebenso Internetsperren, Vorratsdatenspeicherung, Videoüberwachung, Polizeirechte und Maßnahmen gegen Kriminalität und Terrorismus.

Die Novellierung des Bundesdatenschutzgesetzes 2009 erhielt entsprechend viel Aufmerksamkeit. Weitere Novellen sind wahrscheinlich, nicht zuletzt auf Druck der EU. Wahrscheinlich ist zudem eine Neustrukturierung der Datenschutzstellen, da der Europäische Gerichtshof (2010) in einem Vertragsverletzungsverfahren gegen Deutschland eine zu geringe Staatsferne moniert hat. Größere Unabhängigkeit der Datenschutzbeauftragten hätte wohl mehr politischen Aktivismus zur Folge - denkbar auch bei RFID.

Diese Debatten sind inzwischen in alle Bundestagsparteien eingedrungen. Zudem haben sie der 2006 gegründeten Piratenpartei einen hohen Mitgliederzuwachs und erhebliches Medieninteresse beschert und schließlich eine Reihe von Wahlerfolgen.

Es sei daran erinnert, dass es den RFID-Kritikern früh gelang, ihre Anliegen in den parlamentarischen Raum zu bringen - in den Bundestag über die Oppositionsfraktionen FDP, Grüne und Linke, aber auch ins Europäische Parlament und in die deutschen Landtage. Auf dieser Bühne erhalten die Themen Medienaufmerksamkeit und Beachtung der politischen Eliten.

Regierungsmehrheiten können Entscheidungen zwar abblocken, nicht aber die Themenwahrnehmung. Umgekehrt können Regierungen die Thematik von sich aus aufgreifen, auch in den Ländern. Gesetzgeberisch ist der Einfluss der Länder begrenzt, im politischen Agenda-Setting jedoch größer. Zudem können sie auf Landes- und Kommunalebene Regeln für den Einsatz im öffentlichen Sektor schaffen.

\section{Kritik an und im Bibliothekswesen}

Ein Beispiel für landespolitische Aktivitäten ist die Initiative der rheinland-pfälzischen Verbraucherministerin Margit Conrad vom Herbst 2010, die mit dem Landesdatenschutzbeauftragten einen »Verbraucherdialog «zu RFID mit Wirtschafts-, Berufs- und Verbraucherverbänden sowie Behörden ins Leben rief. Sie betonte die Notwendigkeit zur Regulierung von RFID, falls Selbstverpflichtungen der Wirtschaft nicht griffen. RFID sei ohne Transparenz nicht kontrollierbar, wirksame Kontrollen und Sanktionen seien nötig. Ein besonderer Fokus des Daten- und Verbraucherschutzes müsse der Einsatz im öffentlichen Sektor sein, beispielsweise in Bibliotheken (MUFV, 2010). Bemerkenswert ist, dass die von der Ministerin präsentierte RFID-Informationsbroschüre gleich auf zwei Seiten auf Bibliotheken zu sprechen kommt und als "Nachteile« des RFID-Einsatzes benennt: »Bei RFID-Einsatz im Bibliotheks- bzw. Büchereiausweis besteht bei spezieller Ausrüstung der Bibliotheken die Möglichkeit Bewegungsprofile zu erstellen«; »Bei Medienrückgabe allein mittels RFID erfolgt in der Regel keine Kontrolle des Mediums auf Beschädigung « sowie »Stellenabbau durch Automatisierung « (MUFV \& Landesbeauftragterfür den Datenschutz, 2010). Wohlgemerkt, dies ist eine offizielle Regierungspublikation. 
Bei den deutschen Nichtregierungsorganisationen ist festzustellen, dass Bibliotheken bisher kein prioritäres Zielobjekt gewesen sind. Strategisch betrachtet sind Konzerne und Sicherheitsbehörden ein dankbareres Ziel, wenn man Protest- und Medienwirkung sowie Mobilisierungspotenzial einrechnet. Dennoch steht RFID in Bibliotheken unter Beobachtung, beispielsweise beim FoeBuD. Der Verein äußerte sich bereits 2006 in seinem Grundlagenpapier »Unsere Positionen« beunruhigt über die Folgen der Standardisierung von Buchetiketten und Outsourcing von RFID-nahen Dienstleistungen bei Lieferanten sowie mangelnder Transparenz selbst geschlossener Systeme:

»Ein Beispiel für den schon stattfindenden Einsatz von RFID in geschlossenen Systemen ist die Nutzung in Bibliotheken. >Die Früchte des Zorns in Bibliothek X hat einen anderen Code als derselbe Titel in Bibliothek Y. Obwohl RFID-Applikationen heute auf geschlossene Systeme beschränkt sind, wird es große Nachfrage nach standardisierter Etikettierung geben. Verleger zum Beispiel könnten eines Tages Bücher an Bibliotheken und Buchhandlungen ausliefern, die beschreibbare Etiketten haben. Jedes Exemplar von >Die Früchte des Zorns ‘ würde dann einen Teil seines Standard-Warencodes tragen, der mit dem auf jedem anderen Exemplar identisch ist. Die Bibliothek wird in der Lage sein, den restlichen Code anzupassen, so dass er ihren Anforderungen der Inventarkontrolle gerecht wird. Selbst wenn geschlossene Systeme geschlossen bleiben, macht ihr Mangel an Transparenz sie beunruhigend vom Standpunkt der Privatsphäre aus. Weil bestimmte Details über geschlossene Systeme möglicherweise nicht leicht erhältlich sind, hätten Konsumenten große Schwierigkeiten bei der Beschaffung der Informationen, die sie benötigen, um Gefährdungen ihrer Privatsphäre einschätzen und sich schützen zu können.«(FoeBuD, 2006)

Die Organisation benennt eine Reihe von Stadt- und Hochschulbibliotheken, die sich der RFID-Technik bedienen. In der Dokumentation ist ersichtlich, dass FoeBuD derzeit über die Hintergründe der Datenverwendung und Datenspeicherung in Bibliotheken wenig weiß. Dennoch wird an anderer Stelle pauschal behauptet:

»Die Verarbeitung dieser Daten im großen Stil allerdings ermöglicht auch `tolle Interessenprofile auf Knopfdruck: 'Sag mir, was Du liest, und ich sage Dir, was Du denkst (und was Dich vielleicht verdächtig macht oder einen bestehenden Verdacht bestätigt).`Mit RFID in Büchern sind Gedanken nicht mehr frei.«(FoeBuD, 2011)

Kritisch äußert sich FoeBuD zu den Nutzerausweisen, insbesondere die mit Studentenausweisen kombinierte Variante in Hochschulbibliotheken (»die doch eigentlich die >Hüterinnen des freien Geistes s sein sollten«). Auch hier werden zahlreiche Einrichtungen namentlich genannt. Außerdem erinnert die Website daran, dass der »Big Brother Award 2004 für die Videoüberwachung in Hochschulen vergeben wurde.

Immer mehr Universitäten und Fachhochschulen spicken ihre Studierendenausweise mit RFID. Ausgelesen werden die Chips meist in den Bibliotheken und in der Mensa, wenn die Studi-Ausweise in die Nähe des Lesegerätes gehalten werden. 
Fehlen nur noch die RFID-Lese-Antennen in den Türrahmen, dann heißt es »Ich weiß, welches Buch Du liest « - »Den Schein kriegst Du nicht, Du warst ja immer zu spät bei der Vorlesung « - Ernähr Dich mal gesünder, dann schaffst Du auch Deine Prüfung...« usw. (FoeBuD, 2011)

Fachleute mögen diese Einlassungen als banal, unqualifiziert und sachlich falsch einstufen. Allerdings basierte auch die RFID-Kritik an Einzelhandel, Gesundheitseinrichtungen oder Staatsverwaltung auf Informationsdefiziten, Gerüchten und Schreckensszenarien. Fachexpertise der Kritiker entwickelte sich erst mit Recherchen und Aktionserfahrungen. Wesentlich ist, dass das Konfliktthema bereits entdeckt ist und gewisse Öffentlichkeit hat. Der RFID-Einsatz in ihren Ausweisen wird z. B. unter Studierenden und Studentenvertretungen diskutiert, wie sich in Blogs, Foren und Publikationen von Studentenvertretungen leicht feststellen lässt.

Auf der institutionellen Seite der Bibliotheken steht dem keine wirklich intensive Debatte über Datenschutz und Sicherheit gegenüber. Das hat mehrere Gründe: Die RFID-Einführung ist im Bibliothekswesen noch längst nicht flächendeckend und ein junges Thema, bei dem technische Fragen vorherrschen. Es gibt außer den allgemeinen Datenschutzbestimmungen kein RFID-spezifisches Recht, das zum Handeln zwänge. Verch (2007) betont, die rechtliche Bewertung sei von der technischen Ausgestaltung des RFID-Einsatzes abhängig. Allgemein werde eine begrenzte RFID-Technik bevorzugt, die auf passive Funkchips zur Identifizierung von Medien ohne datenschutzsensible Personendatenspeicherung setze, außerdem sei die Funkreichweite gering und spezielle Bibliothekssoftware zum Auslesen notwendig. Diese typische Argumentation habe "zu der vorherrschenden und durchaus bequemen Ansicht beigetragen, dass besondere datenschutzrechtliche Vorgaben beim Einsatz der RFID-Chips in Bibliotheken nicht zu beachten sind «(S. 2). Gleichwohl konstatiert Verch Missbrauchsgefahren, da die RFIDBibliothekstechnik nicht losgelöst von kommerziellen Anwendungen und elektronischen Datenspuren im Alltag betrachtet werden könne. Sie meint:

»In jedem Fall nimmt das Bibliothekswesen eine Vorreiterrolle in der Verbreitung der RFID-Technik in Deutschland ein und sollte dieser besonderen Verantwortung Rechnung tragen, indem es sich eng an datenschutzrechtlichen Leitlinien und ethischen Grundsätzen orientiert.«(Verch, 2007, S. 8)

Verch stellt der entspannten Haltung deutscher Bibliotheken die Kontroversen in den USA gegenüber. Sie verweist beispielsweise auf Protestdemonstrationen von Bürgern wie zur RFID-Einführung bei der San Francisco Public Library 2004, auf die grundsätzliche Ablehnung von RFID in Bibliotheken durch Bürgerinitiativen wie der Electronic Frontier Foundation (EFF) sowie auf die intensive Arbeit der American Library Assocation (ALA). Verch interpretiert die in Amerika verbreitete Sorge um den Schutz der Privatsphäre als Folge einer Politik, die Polizei und Geheimdiensten die Daten von Bibliotheksnutzern zugänglich macht - etwa durch Antiterrorgesetze nach 2001 wie dem Patriot Act. Die US-Bürger seien für das Thema Datenschutz in Bibliotheken besonders sensibilisiert, so Verch (S. 2). 
Der Bibliotheksverband ALA habe - mit Berufung auf seinen Ethikkodex - gegen den Patriot Act Stellung bezogen und in Arbeitsgruppen allgemeine Datenschutzrichtlinien formuliert. Dazu gehören seit 2005 auch eine Verbandsresolution sowie Musterrichtlinien für den Einsatz von RFID, die Sicherheitsvorkehrungen gegen unbefugtes Datenauslesen fordern, den Verzicht auf die Speicherung von Personendaten auf Funkchips, umfassende Information der Bibliotheksnutzer über RFID, die Einführung hauseigener Datenschutzrichtlinien und regelmäßige Überprüfung durch externe Stellen. Vereinzelt sei die ALA von Bibliothekaren wegen ihrer Inflexibilität kritisiert worden. Verch sieht die ALA-Empfehlungen dennoch als Vorbild für Deutschland an. Hilfestellungen zum RFID-Datenschutz und zur RFID-Nutzerinformation seien Aufgaben für Bibliotheksverbände (S. 8).

Teilweise kursieren im Bibliothekswesen bereits Hilfestellungen durch Weitergabe vorbildlicher Bürgerinformationen einzelner Bibliotheken und Verbünde. Nach wie vor ist aber in den Verbänden und Arbeitsgemeinschaften die politische Auseinandersetzung mit RFID eine Randerscheinung. Nur gelegentlich thematisiert es die kleine Schar der Experten für Bibliotheksrecht.

Die fehlende kritische Haltung ist erklärbar: Für Bibliothekare erschließt sich der Nutzen der Technologie unmittelbar. Bibliotheken sind mit Handels- oder Logistikunternehmen vergleichbar, die an liberalen Rahmenbedingungen für die RFID-Einführung interessiert sind. Regulierungsauflagen wären für sie bürokratische Hemmnisse für RFID-Investitionen.

Berufsverbände und Gewerkschaften beschäftigen sich in erster Linie mit den internen Folgen der RFID-Einführung für das Bibliothekspersonal, vom Stellenabbaupotenzial über Gesundheitsschutz und Arbeitsplatzgestaltung bis zur Qualifizierung, von der Mitbestimmung und Mitarbeiterbeteiligung bei RFID-Projekten bis zu Betriebs- und Rahmendienstvereinbarungen. Das ist das vorrangige Interesse der Personal- und Betriebsräte. Eine Totalablehnung kann nicht festgestellt werden. Das Fazit einer Ver.diFachtagung 2009 in Berlin kam zu dem Schluss, RFID biete »mehr Chancen als Risiken« (Ver.di, 2009).

Dieser Fokus schließt den Blick über den Tellerrand nicht aus, wie sich z. B. bei Ver.di zeigt, einer Gewerkschaft, die ihr Mandat durchaus allgemeinpolitisch versteht und sich nicht auf die Funktionen eines »ADAC für Arbeitnehmer« zurückzieht, sondern z. B. auch Verbraucherinteressen einbeziehen will. Ver.di muss sich ohnehin mit RFID beschäftigen, da RFID im Handel eine wichtige Rolle spielt und es im Kontext des schnell wachsenden gewerkschaftlichen Arbeitsgebiets der Mitarbeiterüberwachung und der Forderung nach einem Beschäftigten-Datenschutzgesetz steht. Die Ver.di-Arbeitsgruppe RFID ist daher branchenpolitisch bei den Bundesfachgruppen Groß- und Einzelhandel angesiedelt, die dazu Betriebsrätekonferenzen und Publikationen initiiert hat (Ver.di, 2008).

In der bibliothekarischen Fachdiskussion sind in der Branchenpresse, in Verbänden und bei Fachkongressen kritische Einzelstimmen vernehmbar. Der Arbeitskreis kritischer BibliothekarInnen (Akribie) kam gar zu dem Schluss, die »bibliothekarische Ethik gebietet, 
auf den Einsatz von RFID in öffentlichen Bibliotheken zu verzichten.« (Mahrt-Thomsen, 2010, S. 3). Mit Blick auf Fachkongresse monierte die Akribie-Aktivistin Frauke MahrtThomsen, kritische Nachfragen zu RFID seien »weniger willkommen«, das Interesse liege eher darin, »die schöne neue Funk-Welt [...] in positiven Farben gemalt zu bekommen « (S. 1). Sie beklagt mangelnde Sensibilität für die rechtliche, ethische und politische Problematik. Neben den internen Folgen fürs Personal verweist sie auf mögliche MissbrauchsSzenarien und Sicherheitsrisiken in verbreiteten RFID-Systemen, deren Verschlüsselung von Hackern geknackt werden könnten, aber auch Löcher in der Schutzkette durch die Zusammenarbeit mit Lieferanten, Wartungs- und Support-Dienstleistern. Sie kritisiert, dass der RFID-Einsatz in Bibliotheken die Verbreitung der Technologie legitimiere und Akzeptanz für die RFID-Industrie schaffe - hier müssten sich die Bibliothekare fragen lassen, ob sie den Vertrauensvorschuss der Nutzer nicht missbräuchten, wenn sie allzu bereitwillig auf die RFID-Technik umstiegen (S. 15). Datenschutzbeauftragte hätten es sich »nicht allzu schwer gemacht «, RFID-Anwendungen in Bibliotheken zu genehmigen. Sie hätten kaum eine rechtliche Handhabe und stünden unter dem Druck, als »Bedenkenträger « angefeindet oder in ihren Kompetenzen beschnitten zu werden, wenn sie sich bei Modernisierungsmaßnahmen quer stellten (S. 18).

Diese Positionen sind im Mainstream des Bibliothekswesens kaum verankert. Sie geben jedoch - im Sinne »schwacher Signale« - Konfliktherde wieder, die mit steigender RFIDVerbreitung Politik, Organisationen und Bibliotheksnutzer beschäftigen könnten.

\section{Fazit: Politisches Risikomanagement für RFID-Projekte in Bibliotheken}

Bibliotheken haben bei der Einführung und Entwicklung von RFID-Projekten auf das Potenzial von Konflikten mit ihren Stakeholdern zu achten. Das Projektumfeld beinhaltet Unwägbarkeiten und Bedrohungen. Diese Risiken und die relevanten Akteure mit ihrem Stör- aber auch Unterstützungspotenzial zu erkennen und zu analysieren, ist sowohl für die informelle Lobbyarbeit wie für formale Rechtsetzungsprozesse als auch die Öffentlichkeitsarbeit wichtig. Es gilt, die derzeit »schwachen Signale« zu erfassen und in die eigenen Fachdiskussionen und Strategieprozesse einzubringen.

Derzeit ist das Umfeld für RFID in Bibliotheken insgesamt eher als konfliktarm und freundlich zu bewerten, sieht man einmal von den Haushaltszwängen ab. Bleibt es so? Jedenfalls sollte gegenwärtig das Ziel - in Anschluss an Lobby-Strategieempfehlungen nach van Schendelen (2004, S. 174) - sein, die Lage so zu halten, wie sie ist. Praktisch bedeutet das, den Status quo durch die Gewinnung weiterer Unterstützer abzusichern und die positive Wahrnehmung der RFID-Projekte zu erhalten. Wenn möglich, sollten Entscheidungen über RFID forciert und das Tempo erhöht werden. Dabei sollten politisch brisante Thematiken wie der Datenschutz umsichtig bearbeitet, aber öffentlich zurückhaltend kommuniziert werden.

Sollte das Umfeld unfreundlicher werden, ist die Strategie anzupassen. In einer solchen Lage ist es nach van Schendelen nötig, in den Dialog mit Skeptikern und Kritikern einzutreten und sie soweit wie möglich einzubinden. Die negative Wahrnehmung der Projekte muss eingehegt, ein möglicher Schaden begrenzt werden. Setzt die Politik zu 
Regulierungsaktivitäten an, sollten diese zeitlich gebremst werden. Dabei geht es nicht um Totalblockade, sondern um zeitliche Spielräume für erweiterte Diskussionen und Überzeugungsarbeit, möglichst unter Einbeziehung neuer Verbündeter und an die Interessenlage der Politik angepasster Argumente.

Der nächste Datenschutz-Skandal kommt bestimmt. Ob öffentliche Bibliotheken irgendwann maßgeblich davon betroffen sein werden, ist schwer vorherzusagen. Aber sie gehören zum Staatsapparat und sind schon deshalb ein leichtes Ziel für politische Angriffe. Ihre Technik ist ein Einfallstor für einen symbolischen Streit um gesellschaftliche Akzeptanz von RFID. Die Politik steht unter Druck, den Schutz von Daten und Bürgerrechten durchzusetzen, exemplarisch und symbolisch »im eigenen Haus « - auch und gerade lokal und regional. Gesellschaftlicher Widerstand würde die Bereitschaft der Politik zu hohen RFID-Investitionen bei Bibliotheken absenken.

Überdies haben Bibliotheken eine besondere Klientel, die ihnen einen hohen Vertrauensvorschuss gibt. Enttäuschung und Empörung können umso heftiger ausfallen. Bibliotheksnutzer könnten zum »Wutbürger « mutieren, die gegen moderne Technik und politisch-technokratische Bevormundung rebellieren, sich organisieren und auf die Politik Druck ausüben. Das muss nicht gleich eine nationale Bewegung sein: Auch lokal birgt RFID ein Saatkorn von Misstrauen und Zorn gegen Institutionen, die berechtigte Bürgersorgen nicht ernst nehmen. RFID-Projekte sollten auf Prävention achten.

Die RFID-Debatte wird auf allen politischen Ebenen fortgeführt. Selbstverpflichtungen der Wirtschaft dürften kurzfristig Standards fixieren. Langfristig sind auch gesetzliche Regeln zu erwarten. Dass eine neue Technologie flächendeckend eingeführt wird, ohne einer spezifischen, allmählich engeren Regulierung unterworfen zu werden, ist unwahrscheinlich. Dabei werden nicht nur deutsche und europäische Gesetzgeber aktiv werden, sondern auch die Gerichte. Das wird zu beobachten und auszuwerten sein.

Für die Strategie der Bibliotheken bleibt anzumerken, dass sie mit guter Praxis bei Datenschutz und Informationspolitik Konflikte um RFID überstehen können. Wenn sie den politischen Dialog ernstnehmen, Transparenz zeigen, sich eigene sensible Regeln geben und Ethik-Fragen nicht ausweichen, liegen die Chancen nicht schlecht. Das gilt auch für den Anspruch, per Lobbying künftige Gesetze zu RFID mitgestalten zu wollen - ein Anspruch, den Bibliotheken und ihre Verbände laut und klar erheben sollten. 


\section{Literaturverzeichnis}

American Library Association. (2011). Advocating in a Tough Economy Toolkit. Abgerufen am 17. August 2011 von ALA Issues \& Advocacy: http://www.ala.org/ala/issuesadvocacy/advocacy/ advocacyuniversity/toolkit/index.cfm

Bundesministerium des Innern. (14. Mai 2009). Dritter Bericht des BMI über die Sponsoringleistungen an die Bundesverwaltung. Abgerufen am 29. August 2011 von http://www.bmi.bund.de/SharedDocs/ Downloads/DE/Veroeffentlichungen/dritter_sponsoringbericht.pdf?_blob=publicationFile

Bundesministerium für Wirtschaft und Technologie. (Juli 2007). European Policy Outlook RFID. Abgerufen am 3. August 2011 von http://www.nextgenerationmedia.de/documents/European Policy_Outlook_Paper_RFID_Finale_Version_dt..pdf

Denkler, T. (31. Juli 2007). Der gesponserte Staat. Abgerufen am 12. August 2011 von Sueddeutsche.de: http://www.sueddeutsche.de/politik/bericht-der-bundesregierung-der-gesponserte-staat-1.785742

Deutscher Bibliotheksverband. (14. September 2010). Bibliotheksverband schlägt Alarm: zwei Drittel aller kommunalen Bibliotheken von Einsparungen betroffen, Pressemitteilung. Abgerufen am 2. Juni 2011 von http://www.bibliotheksverband.de/fileadmin/user_upload/DBV/ pressemitteilungen/2010/2010-09-14_PM_Finanzlage_01.pdf

Deutscher Bundestag. (23. Januar 2008). Bericht der Bundesregierung zu den Aktivitäten, Planungen und zu einem möglichen gesetzgeberischen Handlungsbedarf in Bezug auf die datenschutzrechtlichen Auswirkungen der RFID-Technologie, Drucksache 16/7891. Abgerufen am 21. August 2011 von http://dipbt.bundestag.de/dip21/btd/16/078/1607891.pdf

Deutscher Bundestag. (15. August 2011). Dokumentations- und Informationssystem (DIP), Dokumentensuche »RFID«, 16. Wahlperiode. Abgerufen am 15. August 2011 von Deutscher Bundestag: http://dipbt.bundestag.de/dip21.web/searchDocuments/simple_search.do

Deutscher Bundestag. (6. Januar 2010). Unterrichtung des Ausschusses für Bildung, Forschung und Technikfolgenabschätzung: Technikfolgenabschätzung (TA) Zukunftsreport Ubiquitäres Computing, Drucksache 17/405. Abgerufen am 2. August 2011 von http://dipbt.bundestag.de/ dip21/btd/17/004/1700405.pdf

Europäische Kommission. (12. Mai 2009a). Empfehlung zur Umsetzung der Grundsätze der Wahrung der Privatsphäre und des Datenschutzes in RFID-gestützten Anwendungen, K(2009) 3200. Abgerufen am 22. August 2011 von http://eur-lex.europa.eu/LexUriServ/LexUriServ. do?uri=OJ:L:2009:122:0047:0051:DE:PDF

Europäische Kommission. (18. Juni 2009b). Mitteilung der Kommission an das Europäische Parlament, den Rat, den Europäischen Wirtschafts- und Sozialausschuss und den Ausschuss der Regionen vom 18. Juni 2009 über das Internet der Dinge - ein Aktionsplan für Europa (KOM(2009)0278). Abgerufen am 22. August 2011 von http://eur-lex.europa.eu/LexUriServ/LexUriServ. do?uri=COM:2009:0278:FIN:DE:PDF

Europäischer Gerichtshof. (9. März 2010). Urteil des Gerichtshofs (Große Kammer) Vertragsverletzung eines Mitgliedstaats - Richtlinie 95/46/EG - in der Rechtssache C518/07. Abgerufen am 9. August 2011 von http://curia.europa.eu/jurisp/cgi-bin/gettext.pl?where= \&lang=de\&num=79899690C19070518\&doc=T\&ouvert=T\&seance=ARRET

Europäisches Parlament. (15. Juni 2010). Entschließung des Europäischen Parlaments vom 15. Juni 2010 zu dem Internet der Dinge (2009/2224(INI)), P7_TA(2010)0207. Abgerufen am 5. August 2011 von http://www.europarl.europa.eu/sides/getDoc.do?type=TA\&reference=P7-TA-20100207\&language $=\mathrm{DE}$

FoeBuD. (2011). Big Brother Awards. Abgerufen am 23. August 2011 von http://www.bigbrotherawards. de/

FoeBuD. (15. Oktober 2008). Die Gewinnerinnen und Gewinner des Wettbewerbs für ein RFID-Warnlogo. Abgerufen am 23. August 2011 von http://www.foebud.org/rfid/rfid-warnlogo-wettbewerb/diegewinnerinnen-und-gewinner-des-wettbewerbs-fuer-ein-rfid-warnlogo/

FoeBuD. (28. Januar 2006). Schnüffelchips: RFID-Industrie setzt auf PR-Offensive statt auf konstruktiven Dialog. Abgerufen am 10. Juli 2011 von FoeBuD: http://www.foebud.org/rfid/schnueffelchips-rfidindustrie-setzt-auf-pr-offensive-statt-auf-konstruktiven-dialog/

FoeBuD. (29. Dezember 2006). Unsere Positionen. Abgerufen am 25. August 2011 von http://www. foebud.org/rfid/unsere-positionen 
FoeBuD. (2011). Wo gibt es RFID? Abgerufen am 15. August 2011 von http://www.foebud.org/rfid/ wo-gibt-es-rfid

Hamann, G. (19. Januar 2006). Chip, Chip, hurra? Datenschützer kontra Handel - der Streit um die Zukunft der RFID-Funktechnologie eskaliert. Abgerufen am 15. Juli 2011 von Zeit online: http:// www.zeit.de/2006/04/RFID/komplettansicht

Huber, A. (10. August 2007). Koalitionen und Strategische Allianzen: Praxis der Bildung und Steuerung. Berlin: Deutsches Institut für Public Affairs.

Informationsforum RFID. (2009d). »Die Debatte bleibt aktuell«. Abgerufen am 22. August 2011 von RFID Special: Fünf Jahre Informationsforum RFID: http://info-rfid.de/info-rfid/content/e107/ e127/e781/jahresbericht_2009_ger.pdf

Informationsforum RFID. (1. April 2008a). Informationsforum RFID und ORACLE setzen bundesweite RFID-Veranstaltungsreihe für kleine und mittlere Unternehmen fort. Abgerufen am 2. August 2011 von Presseportal.de: http://www.presseportal.de/pm/59187/1164084/informationsforum-rfidund-oracle-setzen-bundesweite-rfid-veranstaltungsreihe-fuer-kleine-und

Informationsforum RFID. (5. Mai 2009). Mittelstandspreis RFID 2009 - jetzt bewerben. Abgerufen am 5. August 2011 von Presseportal.de: http://www.presseportal.de/pm/59187/1399601/ mittelstandspreis-rfid-2009-jetzt-bewerben

Informationsforum RFID. (2011). Positionspapiere 2006-2009. Abgerufen am 29. August 2011 von http://info-rfid.de/publikationen/positionspapiere/index_ger.html

Informationsforum RFID. (2009c). RFID Special: Fünf Jahre Informationsforum RFID. Abgerufen am 22. August 2011 von http://info-rfid.de/info-rfid/content/e107/e127/e781/jahresbericht_2009_ger.pdf

Informationsforum RFID. (Oktober 2008b). RFID zeigt Gesicht! Wettbewerb. Abgerufen am 10. August 2011 von http://info-rfid.de/info-rfid/content/e107/e127/e230/rfid_beileger_logowettbewerb_ger. pdf

Kümmerlen, R. (10. März 2009). Initiative soll RFID voranbringen. Abgerufen am 22. August 2011 von Deutsche Verkehrs-Zeitung: http://www.dvz.de/nc/en/news/alle-news/einzelseite/id/initiativesoll-rfid-voranbringen.html

LobbyControl. (22. September 2005). FIFA und Metro gegen ZDF-Bericht über RFID-Funkchips. Abgerufen am 29. Juli 2011 von LobbyControl: http://www.lobbycontrol.de/blog/index. php/2005/09/fifa-und-metro-gegen-zdf-bericht-ueber-rfid-funkchips/

Mahrt-Thomsen, F. (25. Februar 2010). RFID - moderne Technik mit Tücken. Abgerufen am 22. März 2011 von Staatsbibliothek zu Berlin, Fachtagung der Virtuellen Fachbibliothek Recht und der Arbeitsgemeinschaft für juristisches Bibliotheks- und Dokumentationswesen: http://www.vifarecht.de/fachtagung2010/download/Vortrag_Mahrt-Thomsen_Web.pdf

MUFV \& Landesbeauftragterfür den Datenschutz. (2010). RFID Radiofrequenzidentifikation: Was ist das? Informationsbroschüre. Mainz: Ministerium für Umwelt, Forsten und Verbraucherschutz und Landesbeauftragterfür den Datenschutz Rheinland-Pfalz.

MUFV. (7. September 2010). Conrad und Wagner: »Funktechnologie RFID braucht Kennzeichnung und Transparenz - Broschüre informiert«. Abgerufen am 3. August 2011 von Ministerium für Umwelt, Forsten und Verbraucherschutz: http://www.mulewf.rlp.de/aktuelles/einzelansicht/ archive/2010/september/article/conrad-und-wagner-funktechnologie-rfid-braucht-kennzeichnung-und-transparenz-broschuere-inf/?Fsize=1\&cHash=7d199a7afb9128e23b840ae9b1373d0b

Quack, K. (6. März 2009). EU ergreift die RFID-Initiative. Abgerufen am 19. August 2011 von Computerwoche.de: http://www.computerwoche.de/software/erp/1889319/

Ratzek, W. (2010). So funktioniert Lobbyarbeit - Beispiele aus der Praxis. In W. Ratzek, Lobbyarbeit für Information Professionals: Grundlagen - Beispiele - Empfehlungen (S. 11-36). Bad Honnef: Bock + Herchen.

Rawe, S., \& Schulz, J. (2005). Issues Management. In S. Rawe, M. Althaus, \& M. Geffken, Handlexikon Public Affairs (S. 111-114). Münster und Berlin: Lit.

RFID im Blick. (4. März 2011). Quo Vadis Informationsforum RFID? Abgerufen am 20. August 2011 von RFID im Blick: http://www.marktplatz-rfid-im-blick.de/201103042827/quo-vadisinformationsforum-rfid.html

Stegherr, M. (Dezember 2005a). Die Lobby im Schnellboot. Politik \& Kommunikation, S. 46-48. 
Stegherr, M. (Dezember 2005b). Regulierung zerstört Ansätze. Politik \& Kommunikation , S. 49.

van Schendelen, R. (2004). Machiavelli in Brussels: the art of lobbying the EU. Amsterdam: Amsterdam University Press.

Ver.di. (Juli 2009). Fachtagung Bibliotheken am 12. Juni 2009 in Berlin RFID in Bibliotheken - mehr Chancen als Risiken. Abgerufen am 18. Juli 2011 von http://gemeinden.bb.verdi.de/berlin_-_fb_7/ fk_bibliotheken

Ver.di. (20. August 2008). Radio Frequency Identification (RFID) - menschengerecht gestalten. Abgerufen am 15. Juli 2011 von http://handel.verdi.de/branchenpolitik/rfid

Verch, U. (16. April 2007). Selbstklebend, selbstverbuchend und auch selbstverpflichtend? Rechtliche Rahmenbedingungen für den Einsatz von RFID-Chips in Bibliotheken. Vortrag, Bibliothekskongress Leipzig. Abgerufen am 15. August 2011 von Berufsverband Information Bibliothek: http://www. opus-bayern.de/bib-info/volltexte/2007/305/pdf/verch-leipzig-2007.pdf

von Münchow, A. (Frühjahr 2006). Strategische Allianzen: Effektivere Lobbyarbeit für Verbände und Unternehmen durch Koalitionen. Public Affairs Manager, S. 38-45.

Wiebusch, D. (2005). Strategische Allianzen. In M. Althaus, S. Rawe, \& M. Geffken, Handlexikon Public Affairs (S. 217-220). Münster und Berlin: Lit. 